\title{
Cross-reactivity of the BRAF VE1 antibody with epitopes in axonemal dyneins leads to staining of cilia
}

\author{
Robert T Jones $^{1}$, Malak S Abedalthagafi ${ }^{1}$, Mohan Brahmandam ${ }^{2}$, Edward A Greenfield ${ }^{2}$, \\ Mai P Hoang ${ }^{3}$, David N Louis ${ }^{3}$, Jason L Hornick ${ }^{1}$ and Sandro Santagata ${ }^{1,4}$ \\ ${ }^{1}$ Division of Neuropathology, Department of Pathology, Brigham and Women's Hospital, Harvard Medical \\ School, Boston, MA, USA; ${ }^{2}$ Monoclonal Antibody Core, Dana-Farber Cancer Institute and Dana-Farber/ \\ Harvard Cancer Center, Harvard Medical School, Boston, MA, USA; ${ }^{3}$ Department of Pathology, \\ Massachusetts General Hospital, Harvard Medical School, Boston, MA, USA and ${ }^{4}$ Department of Cancer \\ Biology, Dana-Farber Cancer Institute, Harvard Medical School, Boston, MA, USA
}

\begin{abstract}
Antibodies that recognize neo-epitopes in tumor cells are valuable tools in the evaluation of tissue biopsy or resection specimens. The VE1 antibody that recognizes the V600E-mutant BRAF protein is one such example. We have recently shown that the vast majority of papillary craniopharyngiomas-tumors that arise in the sellar or suprasellar regions of the brain-harbor BRAF V600E mutations. The VE1 antibody can be effective in discriminating papillary craniopharyngioma from adamantinomatous craniopharyngioma, which harbors mutations in CTNNB1 and not BRAF. While further characterizing the use of the VE1 antibody in the differential diagnosis of suprasellar lesions, we found that the VE1 antibody stains the epithelial cells lining Rathke's cleft cysts with very strong staining of the cilia of these cells. We used targeted sequencing to show that Rathke's cleft cysts do not harbor the BRAF V600E mutation. Moreover, we found that the VE1 antibody reacts strongly with cilia in various structures-the bronchial airways, the fallopian tubes, the nasopharynx, and the epididymis-as well as with the flagella of sperm. In addition, VE1 reacts strongly with the cilia of the ependymal lining of the brain and with the cilia-containing microlumens of ependymoma tumors. There is significant sequence homology between the synthetic peptide (amino acid 596-606 of BRAF V600E: GLATEKSRWSG) that was used to generate the VE1 antibody and regions of multiple axonemal dynein heavy chain proteins (eg, DNAH2, DNAH7, and DNAH12). These proteins are major components of the axonemes of cilia and flagella where they drive the sliding of microtubules. In ELISA assays, we show that the VE1 antibody recognizes epitopes from these proteins. A familiarity with the cross-reactivity of the VE1 antibody with epitopes of proteins in cilia is of value when evaluating tissues stained with this important clinical antibody. Modern Pathology (2015) 28, 596-606; doi:10.1038/modpathol.2014.150; published online 21 November 2014
\end{abstract}

'Mutation-specific' antibodies that recognize mutant forms of oncoproteins but not the wild type forms of those proteins are very useful in the evaluation of tumor resection specimens. For example, antibodies that recognize the V600E mutant of BRAF, ${ }^{1,2}$ and the $\mathrm{R} 132 \mathrm{H}$ mutant of IDH1 (refs 3,4) have quickly become part of the clinical armamentarium, assisting pathologists with the diagnostic classification of

Correspondence: Dr S Santagata MD, PhD, Division of Neuropathology, Department of Pathology, Brigham and Women's Hospital, Harvard Medical School, Harvard Institute of Medicine, HIM-921, 77 Avenue Louis Pasteur, Boston, MA 02115, USA. E-mail: ssantagata@partners.org

Received 28 July 2014; revised 12 September 2014; accepted 13 September 2014; published online 21 November 2014 tumors and helping to determine prognosis and predict response to therapeutics. ${ }^{5-7}$ The use of these antibodies is particularly important as the results of genetic testing can still take weeks to report in many institutions. ${ }^{8}$ As these antibodies are so commonly used, it is important to have a thorough understanding of their limitations so as to avoid incorrect interpretations.

We recently demonstrated that papillary craniopharyngioma frequently harbor mutations in $B R A F$, and that the VE1 antibody that recognizes the BRAF V600E-mutant protein can help distinguish craniopharyngioma tumors with wild-type $B R A F$ from those carrying the V600E mutation. ${ }^{9}$ Along with adamantinomatous craniopharyngioma, Rathke's cleft cysts are part of the differential diagnosis that 


\section{Papillary \\ Craniopharyngioma}
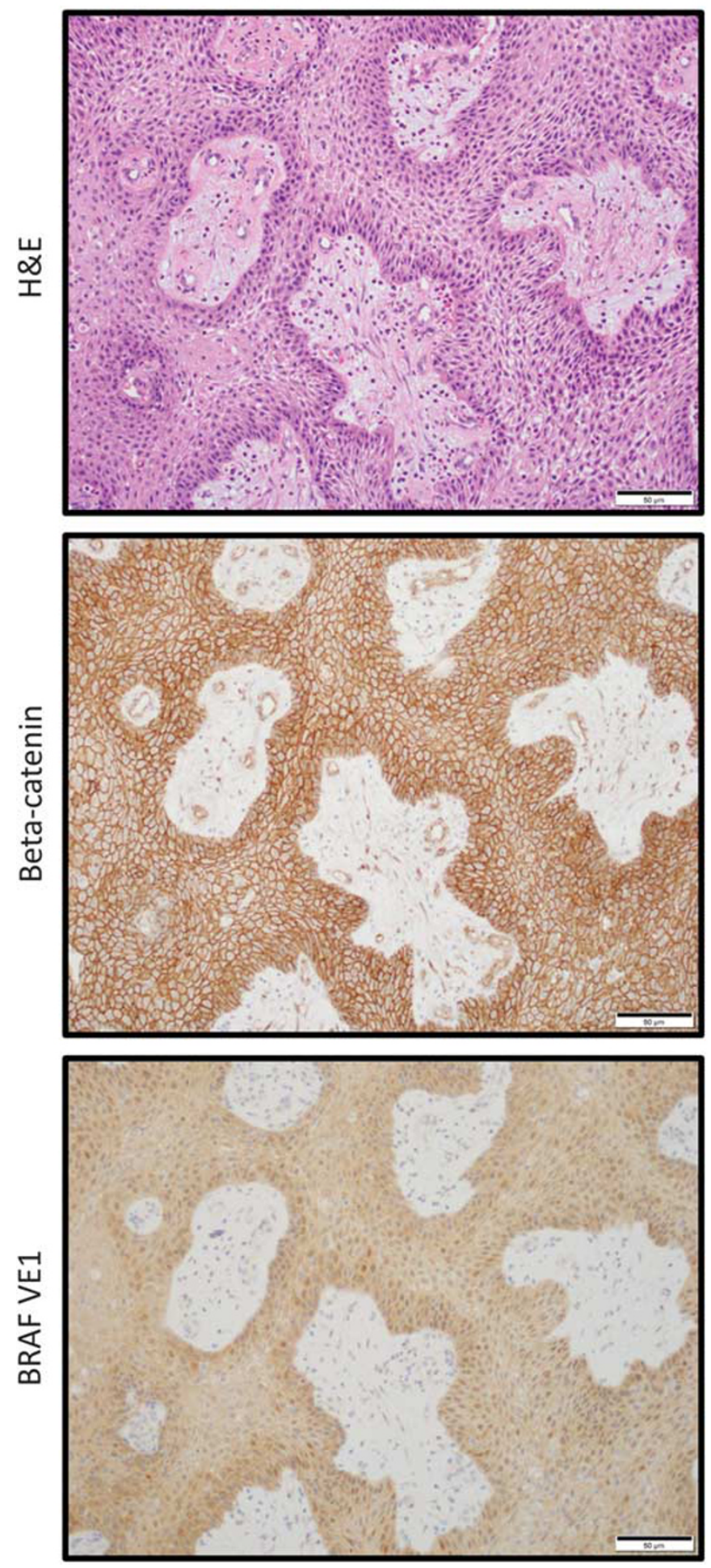

Figure 1 Immunohistochemistry analysis of papillary craniopharyngioma and Rathke's cleft cyst. Images of hematoxylin and eosin (H\&E)-stained sections of both papillary craniopharyngioma and Rathke's cleft cyst are displayed along with images of immunohistochemistry staining for $\beta$-catenin and for BRAF V600E (using the BRAF VE1 mutation-specific antibody). Scale bar, $50 \mu \mathrm{m}$ for papillary craniopharyngioma and $20 \mu \mathrm{m}$ for Rathke's cleft cyst (scale bar in insert is $5 \mu \mathrm{m}$ ). 
neuropathologists contend with when evaluating sellar and suprasellar tumors. ${ }^{10-12}$ To investigate whether Rathke's cleft cysts harbor BRAF V600E mutations, we stained cases with the BRAF VE1 antibody.

\section{Materials and methods}

\section{Tissue and DNA Extraction}

From the archives of Brigham and Women's Hospital (Boston, MA) we retrieved 20 formalin-fixed, paraffin-embedded tissue samples of Rathke's cleft cysts. The study was reviewed and approved by the human subjects institutional review boards of the Dana-Farber Cancer Institute and Brigham and Women's Hospital. Histologic diagnosis was confirmed on all samples by a board-certified neuropathologist (SS). DNA was extracted from tissue shavings or core punches $(1 \mathrm{~mm}$, Miltex, catalog number 33-31AA-P/25) from formalin-fixed, paraffin-embedded tissue blocks.

\section{Immunohistochemistry}

We performed immunohistochemical studies for BRAF V600E expression on $5-\mu \mathrm{M}$-thick sections of formalin-fixed, paraffin-embedded tissue in a Bond 3 automated immunostainer (Leica Microsystems, Bannockburn, IL, USA) using a primary antibody that was designed to recognize the BRAF V600E protein (clone: VE1, 1:100, Spring Bioscience, Pleasanton, CA). We deparaffinized the tissue sections using Bond Dewax solution and used Leica Polymer Refine Kit for the diaminobenzidine staining. For $\beta$-catenin staining we performed antigen retrieval in a pressure cooker in citrate buffer $(\mathrm{pH}$
6.0, 1:1000 dilution). The sections were incubated for $45 \mathrm{~min}$ in primary antibody (BD Pharmigen, catalog number 610154, mouse-monoclonal, clone: 14) followed by Dako anti-mouse-horseradish peroxidase for $30 \mathrm{~min}$ at room temperature. Cases with nuclear staining were scored as positive and membranous staining was scored as negative.

\section{Sequenom Genotyping of BRAF Mutations}

We used mass spectrometric genotyping based on the Sequenom MassARRAY technology (Sequenom, San Diego, CA) using a multi-base homogenous mass-extend (hME) as previously described ${ }^{13}$ to assay DNA extracted from Rathke's cleft cysts for mutations in BRAF.

\section{Expression Profiling}

We normalized expression profiling data from publicly available brain tumor data sets collected using the Affymetrix Human Genome U133 Plus 2.0 Array Platform [HG-U133_Plus_2] and cataloged in GEO (Gene Expression Omnibus) using methods previously described. ${ }^{14}$ The data sets include the following series: GSE16581 (meningioma), GSE34824 (pediatric), GSE19404 (CNS primitive neuroectodermal tumors), GSE35493 (atypical teratoid rhabdoid tumors/ATRT, medulloblastoma, normal brain structures, and primitive neuroectodermal tumor), GSE34771 (CNS lymphoma), GSE36245 (adult glioblastoma), GSE33331 (adult astrocytoma), GSE16155 (ependymoma), and GSE5675 (pilocytic astrocytoma). Heat maps were generated using Genepattern tools (Broad Institute).

Table 1 Characterization of Rathke's cleft cyst specimens by IHC and targeted genotyping

\begin{tabular}{|c|c|c|c|c|}
\hline Case & Ciliated (yes or no) & $B R A F$ VE1 IHC & B-Catenin IHC & BRAF V600E hME \\
\hline RCC1 & Yes & Cilia and cell body & Membrane & Negative \\
\hline RCC2 & Yes & Cilia and cell body & Membrane & Negative \\
\hline RCC3 & Yes & Cilia and cell body & Membrane & Negative \\
\hline RCC4 & Yes & Cilia and cell body & Membrane & Negative \\
\hline RCC5 & Yes & Cilia and cell body & $\mathrm{n} / \mathrm{a}$ & $\mathrm{n} / \mathrm{a}$ \\
\hline RCC6 & Yes & Cilia and cell body & Membrane & Negative \\
\hline RCC7 & No & Negative & Membrane & Negative \\
\hline RCC8 & Yes & Cilia and cell body & Membrane & Negative \\
\hline RCC9 & Yes & Cilia and cell body & Membrane & Negative \\
\hline RCC10 & Yes & Cilia and cell body & Membrane & Negative \\
\hline RCC11 & Yes & Cilia and cell body & Membrane & Negative \\
\hline RCC12 & Yes & Cilia and cell body & Membrane & Negative \\
\hline RCC13 & Yes & Cilia and cell body & Membrane & Negative \\
\hline RCC14 & No & Negative & Membrane & Negative \\
\hline RCC15 & No & Negative & Membrane & Negative \\
\hline RCC16 & Yes & Cilia and cell body & Membrane & Negative \\
\hline RCC17 & No & Negative & Membrane & Negative \\
\hline RCC18 & Yes & Cilia and cell body & Membrane & Negative \\
\hline RCC19 & Yes & Cilia and cell body & $\mathrm{n} / \mathrm{a}$ & Negative \\
\hline RCC20 & No & Negative & Membrane & Negative \\
\hline
\end{tabular}

Abbreviations: hME, homogenous mass-extend; IHC, immunohistochemistry; n/a, not available. 

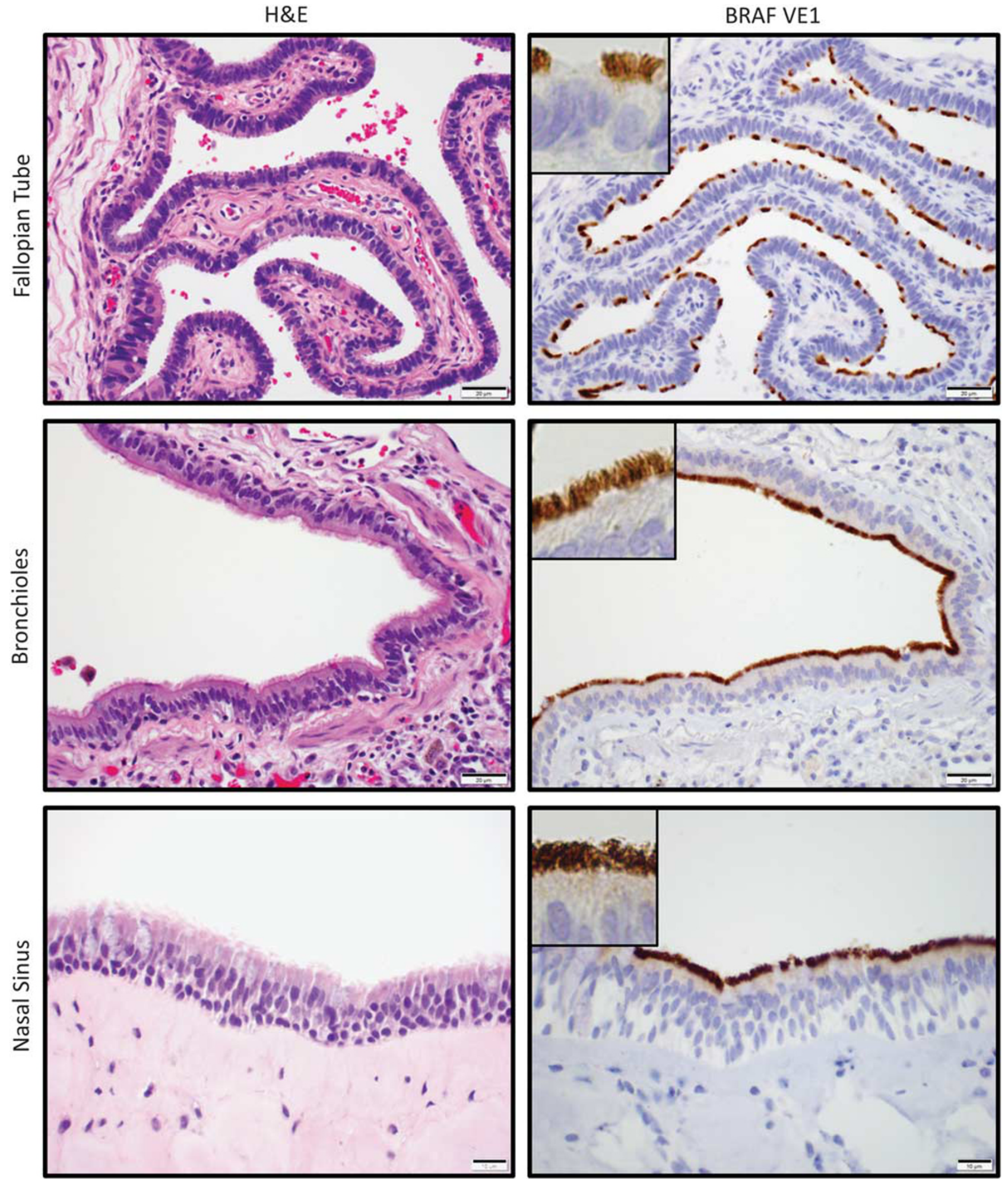

Figure 2 Immunohistochemistry analysis of ciliated epithelium. Images of hematoxylin and eosin (H\&E)-stained sections and images of immunohistochemistry for BRAF V600E (using the BRAF VE1 antibody) of fallopian tube, bronchioles, and sinonasal respiratory epithelium. Scale bar, $20 \mu \mathrm{m}$. 


\section{Indirect ELISA for Cilia Peptides}

Polysterene enzyme immunoassay/radio immunoassay microplates (Costar, catalog number 9018) were coated and kept overnight at $4{ }^{\circ} \mathrm{C}$ with $100 \mu \mathrm{l}$ of various concentrations of peptides $(1000,500,250$, $125,62.5,31.3,15.6,7.8,3.9,2.0$, and $1.0 \mathrm{ng} / \mathrm{ml}$ corresponding to peptide amounts per well of 100 , $50,25,12.5,6.3,3.1,1.6,0.8,0.4,0.2$, and $0.1 \mathrm{ng}$, respectively); see Figure 6 for details of peptide sequence. Wells were blocked with $0.5 \%$ bovine serum albumin in Tris-buffered saline with Tween for $1 \mathrm{~h}$ at room temperature. A dilution of 1:5000 of anti-BRAF antibody (Spring Bioscience, catalog number E19290, clone VE1) was used. The plate was incubated with this primary antibody for $90 \mathrm{~min}$ at room temperature and then washed three times with Tris-buffered saline with Tween. A 1:5000 dilution of goat-anti-mouse-horseradish peroxidase $(\mathrm{H}+\mathrm{L})$ secondary antibody was then added. Incubation with the secondary antibody was for $1 \mathrm{~h}$ at room temperature.
The wells were washed four times with Tris-buffered saline with Tween. Development was with $3,3^{\prime}, 5,5^{\prime}$ Tetramethylbenzidine substrate and the reaction was stopped with $2 \mathrm{~N} \mathrm{H}_{2} \mathrm{SO}_{4}$ after $2 \mathrm{~min}$. Plates were then read at $450 \mathrm{~nm}$ in a spectrophotometer.

\section{Results}

\section{VE1 Antibody Staining of the Cilia of Rathke's Cleft Cysts in the Absence of BRAF V600E Mutations}

Similar to the staining pattern seen in papillary craniopharyngioma, $\beta$-catenin was localized to the membrane of the cyst lining cells of Rathke's cleft cysts (Figure 1 and Table 1) supporting an absence of activating mutations in CTNNB1. To investigate whether Rathke's cleft cysts harbor BRAF V600E mutations, we stained 20 Rathke's cleft cysts with the BRAF VE1 antibody (Table 1). As previously reported, we saw strong staining of pituitary cells

Table 2 BRAF VE1 staining of various human tissues with ciliated cells and sperm

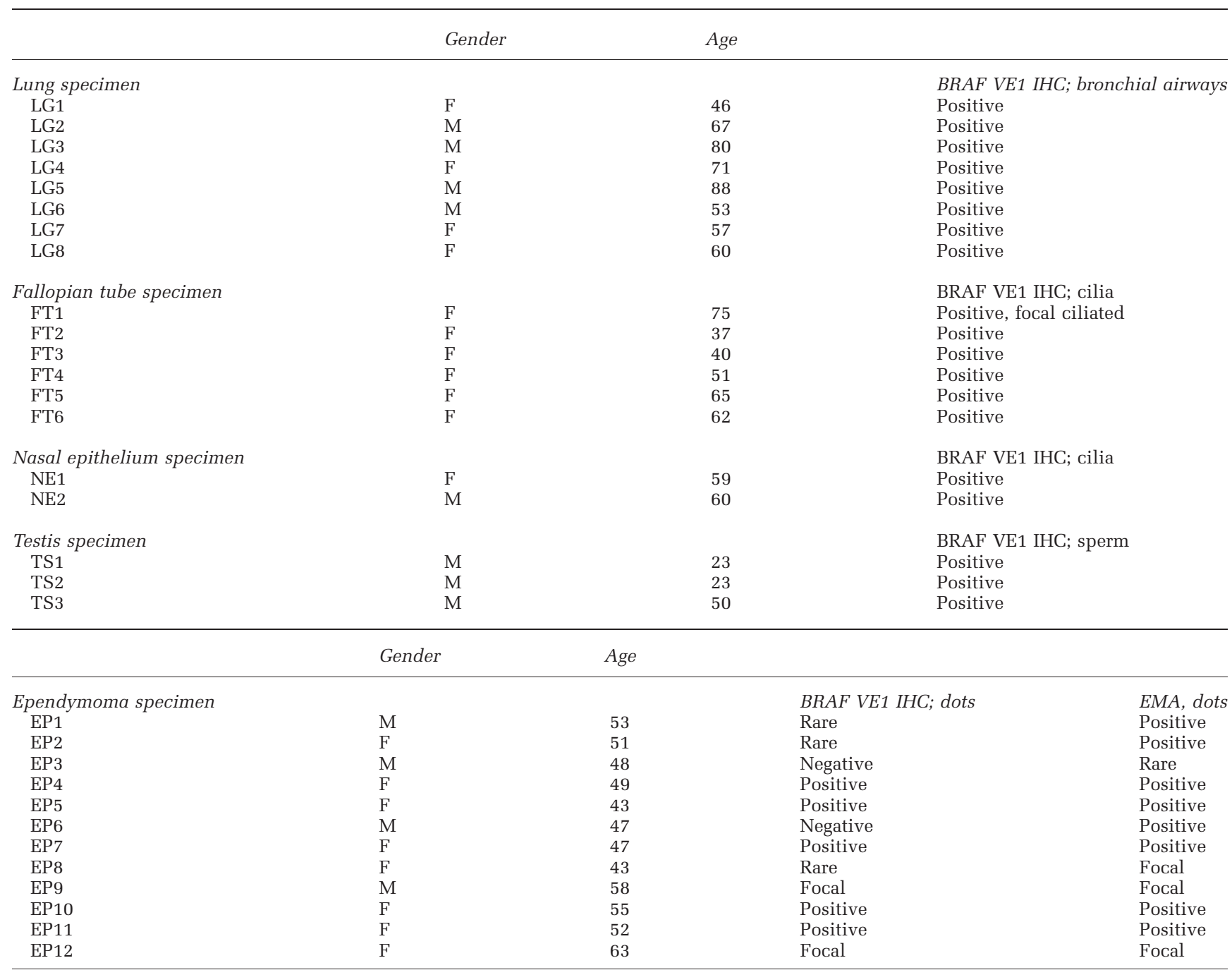

Abbreviations: EMA, epithelial membrane antigen; F, female; IHC, immunohistochemistry; M, male. 
that were incidentally resected along with the cysts. ${ }^{15}$ Although cells that do not carry BRAF V600E mutations should show no reactivity with this antibody, we found strong staining in the epithelial cells of Rathke's cleft cysts (Figure 1). Although the tumor cells of papillary craniopharyngioma have a diffuse nuclear and cytoplasmic staining pattern (Figure 1), we saw intense staining of the cilia of the cyst lining cells and a more modest level of staining of the cell bodies of these epithelial cells (Figure 1). In regions of these cysts that had undergone squamous metaplasia, we found strong staining of the cilia of residual ciliated cells and of the cytoplasm of these ciliated cells, as well as of the cytoplasm of neighboring squamous cells that were not overly ciliated (Supplementary Figure S1). In five of the 20 Rathke's cleft cyst cases, the cells lacked cilia entirely and no staining of the epithelium was seen whether there was squamous metaplasia present or attenuated/cuboidal cells (Table 1).

We performed Sequenom-SNP genotyping based targeted sequencing (hME) using DNA extracted from 19 of the 20 Rathke's cleft cysts (Table 1), to investigate whether these cysts harbored genetic mutations that would encode the BRAF V600E protein. ${ }^{9,13}$ This method has the ability to detect mutations at an allelic fraction of $<5 \%$. Despite the strong staining with the BRAF VE1 antibody, we did not detect the genetic mutation encoding BRAF V600E in any of the Rathke's cleft cysts (Table 1).

\section{VE1 Antibody Staining of the Cilia of Various Tissues}

We next used the VE1 antibody to stain a series of human tissues that have ciliated cells (Figure 2). We noted strong staining of the cilia from fallopian tube $(n=6)$, lung bronchial airways $(n=8)$, and nasopharyngeal airways $(n=2)$ (Table 2). The cell bodies of the ciliated cells had weaker staining. We also noted very strong staining of the cilia of a mucocele of the paranasal sinuses (Supplementary Figure S2). As cilia and flagella have a similar protein
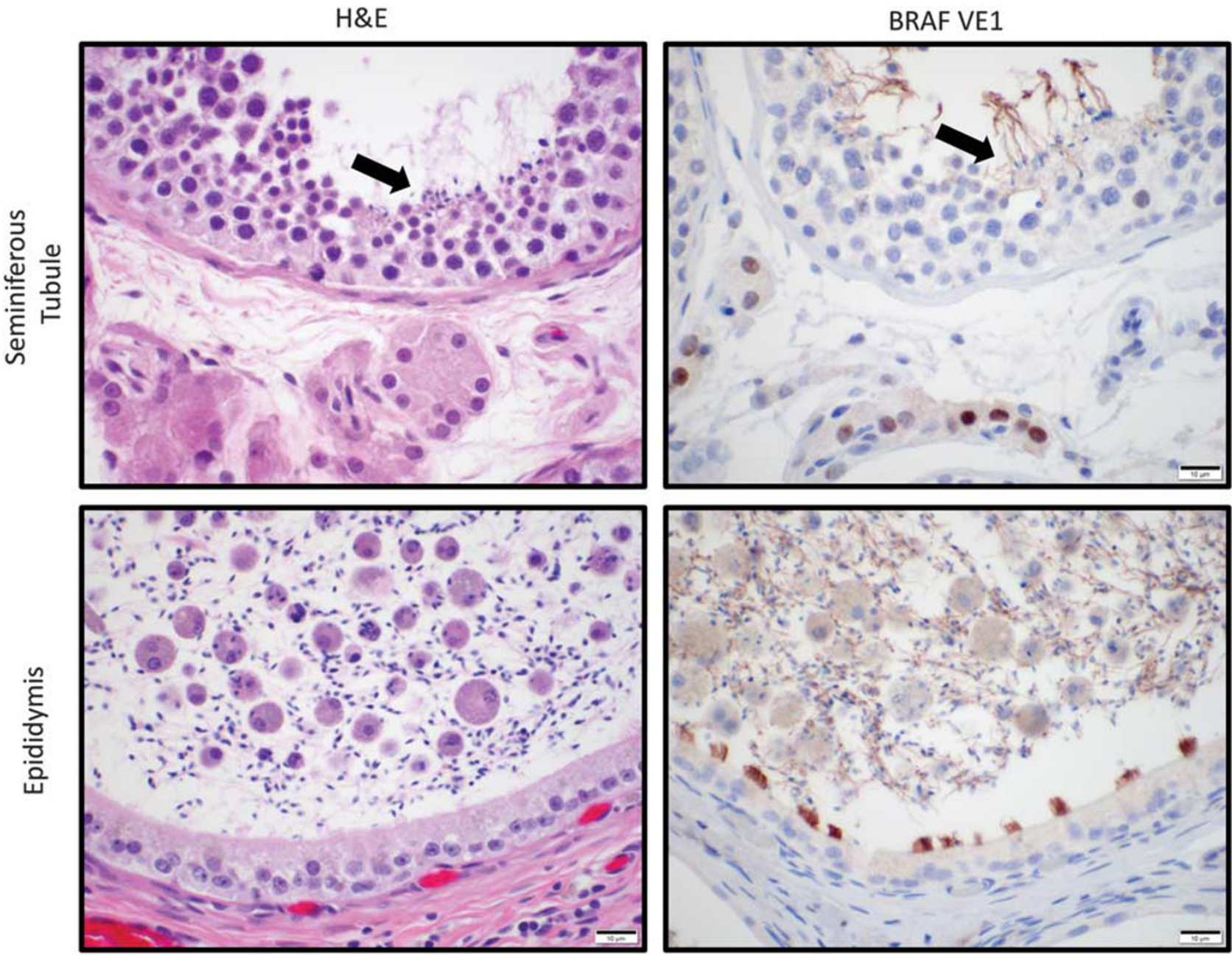

Figure 3 Immunohistochemistry analysis of seminiferous tubules and epididymis. Images of hematoxylin and eosin (H\&E)-stained sections and images of immunohistochemistry for BRAF V600E (using the BRAF VE1 antibody) of seminiferous tubules with mature spermatids and of the epididymis. Leydig cells are seen in the interstitium of the testis. Scale bar, $10 \mu \mathrm{m}$. 
composition and organization, we investigated whether the flagella of mature spermatozoa would also be stained by the VE1 antibody (testis and epididymis $n=3$; Table 2). Indeed, we observed that the VE1 antibody strongly stained the flagella of mature sperm in the seminiferous tubules and in the

\section{Ependymal Lining}

BRAF VE1

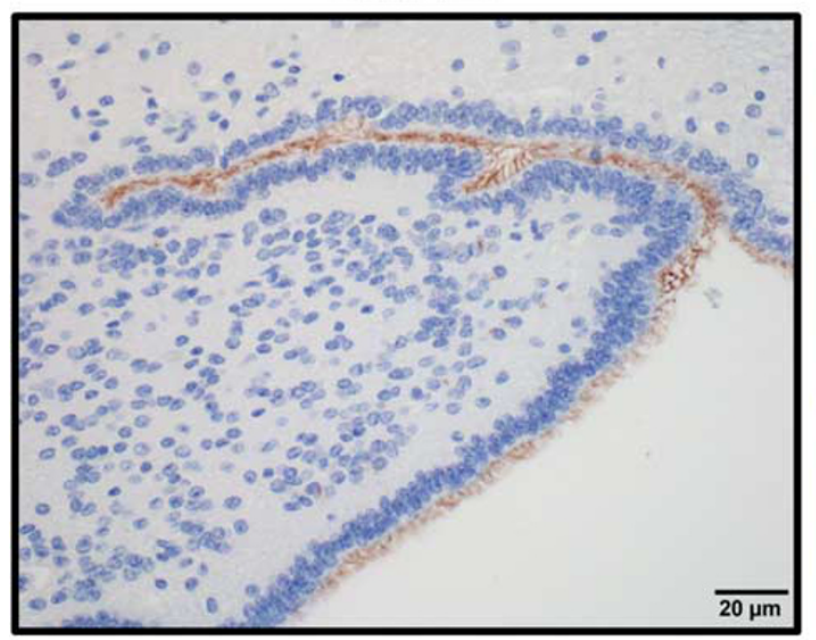

Ependymoma

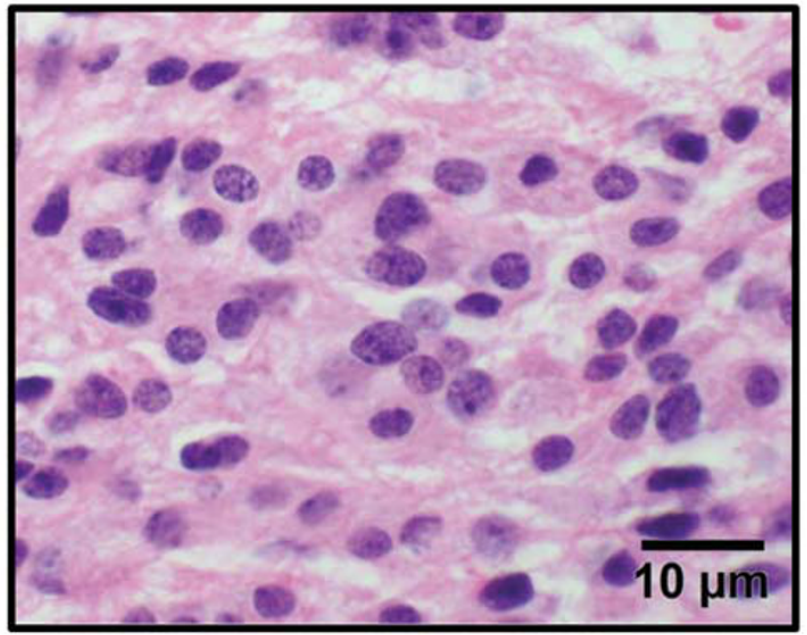

$\underset{\varpi}{\varpi}$

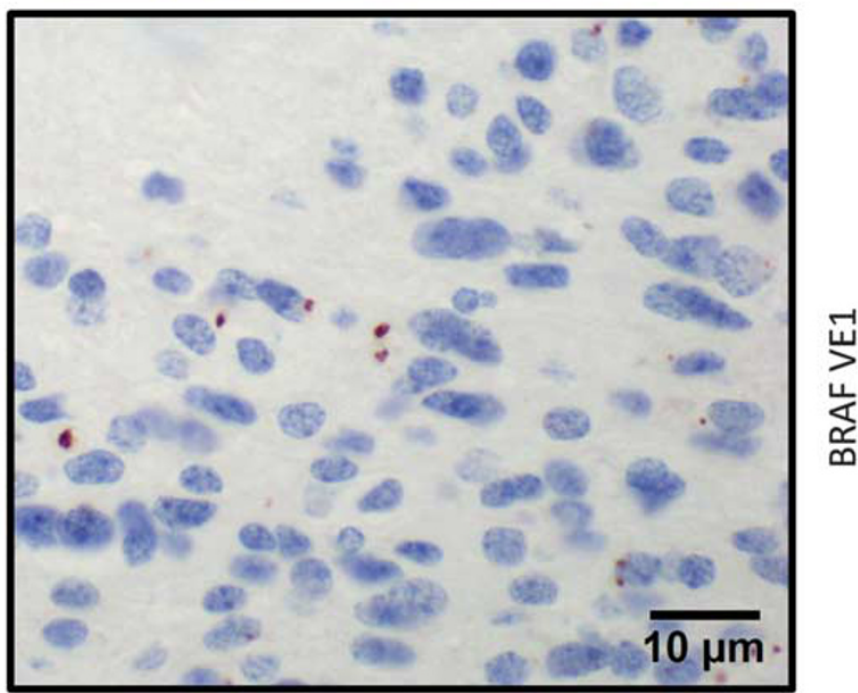

CCDC164

DNAL1

DNAL4

DNAI1

DNALI1

DNAAF1

DNAH1

DNAH2

DNAH3

DNAH5

DNAH6

DNAH7

DNAH8

DNAH9

DNAH10

DNAH11

DNAH12

DNAH14
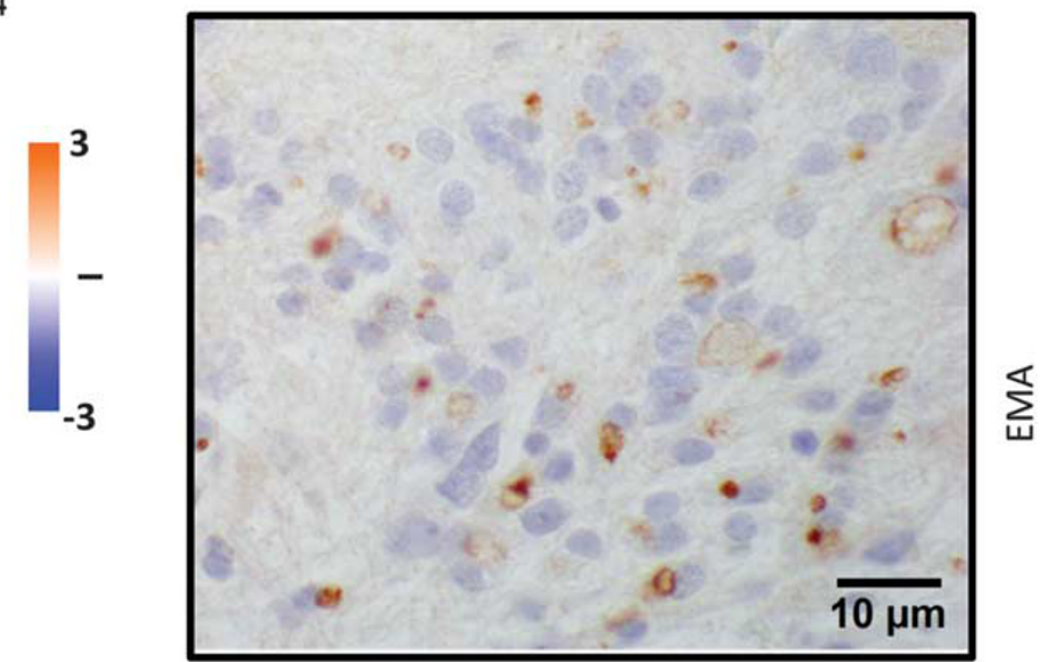
epididymis (Figure 3), as well as the cilia of the epididymis (Figure 3 ). In addition, we noted strong nuclear staining of Leydig cells in the interstitium of the testis, suggesting the presence of other crossreactive epitopes in those cells.

\section{Ependymomas Express Genes that Encode Axonemal Proteins and the Microlumen of Ependymomas are Stained by the VE1 Antibody}

Next, we stained autopsy and neurosurgical resection specimens of adult and pediatric brain that contained ependymal lining $(n=3)$ (Figure 4). Once again, we observed strong staining of the cilia. We analyzed publicly available expression profiling data from a panel of human brain tumors (Supplementary Table S1). Of the 19 ependymoma in this panel, about half (10 of 19 cases) demonstrated moderate expression of genes encoding axonemal proteins, thereby supporting that these tumor cells continue to express the proteins that comprise cilia. Background levels were seen for the other ependymoma samples. In a heat map, we show that genes that encode axonemal proteins are wellexpressed in ependymoma compared with a range of other brain tumors, including astrocytomas, atypical teratoid rhabdoid tumors, and medulloblastoma among others (Figure 4). Nearly half of 12 randomly selected ependymoma formalin-fixed, paraffinembedded samples (5 of 12; Table 2) from our tissue archives showed punctate staining corresponding to the cilia containing microlumens of ependymoma (Figure 4). During clinical diagnosis, punctate staining with epithelial membrane antigen is used to support the diagnosis of ependymoma. In all cases, the punctate staining for epithelial membrane antigen was, however, much stronger than the punctate dots observed with VE1 staining (Figure 4).

\section{The VE1 Antibody Recognizes Epitopes in Axonemal Dyneins}

We next used the BLAST (Basic Local Alignment Search Tool) ${ }^{16}$ to perform a standard protein-protein homology search of the human proteome using the sequence of the peptide immunogen (FGLATEKSRWSG) that had been used to generate the BRAF VE1 antibody (results in Table 3). This peptide demonstrated strong similarity to regions of multiple axonemal dynein heavy chain proteins, including DNAH2, DNAH5, DNAH7, and DNAH12 (Figure 5 and Table 3). In an ELISA assay, we tested the ability of the VE1 antibody to recognize peptides containing the region of interest of wild-type (BRAF_wt) and V600E mutant BRAF (BRAF_mut), as well as homologous regions of several axonemal dyneins (Figure 6). The VE1 antibody robustly recognized the peptide derived from the V600E mutant of BRAF and recognition was minimal for the wild-type BRAF sequence (Figure 6). As predicted by the sequence homology and the strong staining of cilia in tissue sections, peptides with wild-type sequences from the three axonemal dynein heavy chain proteins that we tested (DNAH2, DNAH7, and DNAH12) were all recognized by the VE1 antibody. The relative affinity for the peptide derived from DNAH12 (DNAH12_wt) was very similar to that of peptide from the V600E mutant of BRAF (BRAF_mut). Recognition of the DNAH7-derived peptide (DNAH7_wt) was robust but less than that for the DNAH12-derived peptide. Binding of the VE1 antibody to the DNAH2-derived peptide was detected, but was the weakest of the three axonemal derived peptides tested. When the glutamates that align with the E600 residue in mutant BRAF were changed to valines in the axonemal derived peptides (DNAH2_mut, DNAH7_mut, and DNAH12_mut), recognition by the VE1 antibody was reduced to background levels, similar to the level of recognition of the wild-type BRAF peptide (Figure 6).

\section{Discussion}

Mutation-specific antibodies are valuable tools in clinical practice. An understanding of the limitations of these tools, particularly their cross-reactivity with unintended proteins, is however of significant importance so that the appropriate interpretation of staining results can be rendered. In this work we show that the VE1 antibody that was generated to recognize the BRAF V600E mutant protein also recognizes epitopes in multiple axonemal dynein heavy chain proteins and that the recognition is highly dependent on a single glutamate residue in these axonemal proteins. In most tissues, these proteins are predominantly restricted to the cilia lining the luminal surface of epithelial cells so the cross-reactivity should likely not confound diagnosis. However, moderate staining of the cytoplasm of ciliated cells is also seen in many

Figure 4 Expression of axonemal proteins in the cilia of ependymal lining and in the microlumens of ependymoma. Images of immunohistochemistry for BRAF V600E (using the BRAF VE1 antibody) of the ependymal lining of the human brain. Scale bar, $20 \mu \mathrm{m}$. Images of hematoxylin and eosin (H\&E)-stained sections of a WHO grade II ependymoma and of immunohistochemistry for BRAF V600E (using the BRAF VE1 antibody) and for epithelial membrane antigen. Both stains show punctate dot-like staining. Scale bar, $10 \mu \mathrm{m}$. Heat map of differential expression of axonemal genes in various brain tumors and normal brain tissues $(n=350$ samples). Columns represent each tissue type and are an average of all specimens of the listed tissue classes (Supplementary Table S1). Gene expression data were obtained from public data deposited in the Gene Expression Omnibus (GEO) and were normalized (see Materials and Methods for series information). Expression levels are colored orange for high intensities and blue for low intensities (scale bar presented in low right corner; log2-fold change). 


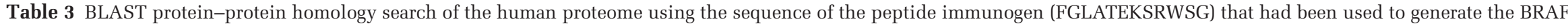
VE1 antibody

\begin{tabular}{|c|c|c|c|c|c|c|}
\hline Description & Max score & Total score & Query cover & E-value & Identity & Accession \\
\hline RAF proto-oncogene serine/threonine-protein kinase & 35 & 35 & $100 \%$ & 0.001 & $92 \%$ & NP_002871.1 \\
\hline Serine/threonine-protein kinase A-Raf & 32.5 & 32.5 & $100 \%$ & 0.008 & $83 \%$ & NP_001243125.1 \\
\hline$\alpha$-Ketoglutarate-dependent dioxygenase alkB homolog 7 & 26.5 & 26.5 & $58 \%$ & 0.59 & $100 \%$ & NP_115682.1 \\
\hline Dynein heavy chain 2, axonemal (DNAH2 & 24 & 24 & $75 \%$ & 4 & $78 \%$ & NP_065928.2 \\
\hline Zinc finger protein $518 \mathrm{~A}$ & 23.1 & 23.1 & $58 \%$ & 7.4 & $86 \%$ & NP 001265455.1 \\
\hline Dynein heavy chain 12, axonemal (DNAH12) & 23.1 & 35.2 & $75 \%$ & 7.4 & $78 \%$ & NP 001278590.1 \\
\hline Dynein heavy chain 7, axonemal (DNAH7) & 23.1 & 23.1 & $83 \%$ & 7.4 & $70 \%$ & NP_061720.2 \\
\hline BTB/POZ domain-containing protein KCTD4 & 22.3 & 22.3 & $75 \%$ & 14 & $80 \%$ & NP_940686.2 \\
\hline Anion exchange protein 3 & 22.3 & 22.3 & $75 \%$ & 14 & $80 \%$ & NP_005061.2 \\
\hline Dynein heavy chain 5 , axonemal (DNAH5) & 22.3 & 49.8 & $75 \%$ & 14 & $78 \%$ & NP_001360.1 \\
\hline Olfactory receptor $9 \mathrm{Q}^{2}$ & 21.4 & 21.4 & $50 \%$ & 26 & $83 \%$ & NP_001005283.1 \\
\hline Armadillo repeat-containing protein 4 & 21.4 & 21.4 & $58 \%$ & 26 & $86 \%$ & NP_-060546.2 \\
\hline Platelet-activating factor acetylhydrolase IB subunit $-\beta$ & 21 & 21 & $41 \%$ & 36 & $100 \%$ & NP_001171675.1 \\
\hline RAB6-interacting golgin & 21 & 21 & $41 \%$ & 36 & $100 \%$ & NP_001139511.1 \\
\hline high-affinity cGMP-specific 3',5'-cyclic phosphodiesterase 9A & 21 & 21 & $50 \%$ & 37 & $100 \%$ & NP_001001576.1 \\
\hline Major facilitator superfamily domain-containing protein 8 (Homo sapiens) & 21 & 21 & $66 \%$ & 37 & $64 \%$ & NP_689991.1 \\
\hline MYELOPEROXIDASE precursor (H. sapiens) & 21 & 21 & $83 \%$ & 37 & $64 \%$ & NP_000241.1 \\
\hline Colorectal mutant cancer protein isoform 2 (H. sapiens) & 21 & 21 & $66 \%$ & 37 & $75 \%$ & NP_002378.1 \\
\hline PREDICTED: colorectal mutant cancer protein isoform X1 (H. sapiens) & 21 & 21 & $66 \%$ & 37 & $75 \%$ & XP_005272048.1 \\
\hline PREDICTED: colorectal mutant cancer protein isoform X2 (H. sapiens) & 21 & 21 & $66 \%$ & 37 & $75 \%$ & $\mathrm{XP}_{0} 006714680.1$ \\
\hline Colorectal mutant cancer protein isoform 1 (H. sapiens) & 21 & 21 & $66 \%$ & 37 & $75 \%$ & NP_001078846.1 \\
\hline F-box only protein 46 (H. sapiens) & 20.6 & 35.6 & $91 \%$ & 51 & $67 \%$ & NP_001073938.1 \\
\hline NACHT, LRR, and PYD domains containing protein 7 isoform 2 (H. sapiens) & 20.6 & 20.6 & $58 \%$ & 51 & $86 \%$ & NP_996611.2 \\
\hline PREDICTED: NACHT, LRR, and PYD domains containing protein 7 isoform X4 (H. sapiens) & 20.6 & 20.6 & $58 \%$ & 51 & $86 \%$ & XP_006723140.1 \\
\hline NACHT, LRR, and PYD domains containing protein 7 isoform 1 (H. sapiens) & 20.6 & 20.6 & $58 \%$ & 51 & $86 \%$ & NP_631915.2 \\
\hline PREDICTED: codanin-1 isoform X3 (H. sapiens) & 20.6 & 20.6 & $50 \%$ & 51 & $100 \%$ & XP_005254235.1 \\
\hline PREDICTED: NACHT, LRR, and PYD domains-containing protein 7 isoform X1 (H. sapiens) & 20.6 & 20.6 & $58 \%$ & 51 & $86 \%$ & XP_006726370.1 \\
\hline NACHT, LRR, and PYD domains containing protein 7 isoform 3 (H. sapiens) & 20.6 & 20.6 & $58 \%$ & 51 & $86 \%$ & NP_001120727.1 \\
\hline NACHT, LRR, and PYD domains containing protein 2 isoform 3 (H. sapiens) & 20.6 & 20.6 & $58 \%$ & 51 & $86 \%$ & NP_001167554.1 \\
\hline NACHT, LRR, and PYD domains containing protein 2 isoform 2 (H. sapiens) & 20.6 & 20.6 & $58 \%$ & 51 & $86 \%$ & NP_001167553.1 \\
\hline PREDICTED: integrin alpha-10 isoform X11 (H. sapiens) & 20.6 & 20.6 & $58 \%$ & 51 & $86 \%$ & XP_006711659.1 \\
\hline NACHT, LRR, and PYD domains containing protein 2 isoform 1 (H. sapiens) & 20.6 & 20.6 & $58 \%$ & 51 & $86 \%$ & NP_-060322.1 \\
\hline PREDICTED: NACHT, LRR, and PYD domains-containing protein 7 isoform X1 & 20.6 & 20.6 & $58 \%$ & 51 & $86 \%$ & XP_006723137.1 \\
\hline PREDICTED: codanin-1 isoform X2 (H. sapiens) & 20.6 & 20.6 & $50 \%$ & 51 & $100 \%$ & XP_005254234.1 \\
\hline PREDICTED: integrin $\alpha-10$ isoform X5 (H. sapiens) & 20.6 & 20.6 & $58 \%$ & 51 & $86 \%$ & XP_005277493.1 \\
\hline PREDICTED: integrin $\alpha$-10 isoform X4 (H. sapiens) & 20.6 & 20.6 & $58 \%$ & 51 & $86 \%$ & XP_005277492.1 \\
\hline PREDICTED: integrin alpha-10 isoform X3 (H. sapiens) & 20.6 & 20.6 & $58 \%$ & 51 & $86 \%$ & $\mathrm{XP}_{-} 005277491.1$ \\
\hline PREDICTED: integrin $\alpha-10$ isoform X2 (H. sapiens) & 20.6 & 20.6 & $58 \%$ & 51 & $86 \%$ & XP_005277490.1 \\
\hline Integrin $\alpha-10$ precursor (H. sapiens) & 20.6 & 20.6 & $58 \%$ & 51 & $86 \%$ & NP_003628.2 \\
\hline PREDICTED: integrin $\alpha-10$ isoform X1 (H. sapiens) & 20.6 & 20.6 & $58 \%$ & 51 & $86 \%$ & XP_005277489.1 \\
\hline Codanin-1 (H. sapiens) & 20.6 & 20.6 & $50 \%$ & 51 & $100 \%$ & NP_612486.2 \\
\hline PREDICTED: codanin-1 isoform X1 (H. sapiens) & 20.6 & 20.6 & $50 \%$ & 51 & $100 \%$ & XP_005254233.1 \\
\hline HEAT repeat-containing protein 5A (H. sapiens) & 20.6 & 49 & $66 \%$ & 51 & $86 \%$ & NP_056288.2 \\
\hline PREDICTED: pericentrin isoform X6 (H. sapiens) & 20.6 & 61.1 & $75 \%$ & 51 & $86 \%$ & XP_005261186.1 \\
\hline PREDICTED: pericentrin isoform X5 (H. sapiens) & 20.6 & 61.1 & $75 \%$ & 51 & $86 \%$ & $\mathrm{XP}_{-} 005261185.1$ \\
\hline PREDICTED: pericentrin isoform X4 (H. sapiens) & 20.6 & 61.1 & $75 \%$ & 51 & $86 \%$ & XP_005261184.1 \\
\hline PREDICTED: pericentrin isoform X3 (H. sapiens) & 20.6 & 61.1 & $75 \%$ & 51 & $86 \%$ & $\mathrm{XP}_{-} 005261183.1$ \\
\hline Pericentrin (H. sapiens) & 20.6 & 61.1 & $75 \%$ & 51 & $86 \%$ & NP_006022.3 \\
\hline PREDICTED: pericentrin isoform X2 (H. sapiens) & 20.6 & 61.1 & $75 \%$ & 51 & $86 \%$ & XP_005261182.1 \\
\hline PREDICTED: pericentrin isoform X1 (H. sapiens) & 20.6 & 61.1 & $75 \%$ & 51 & $86 \%$ & XP_005261181.1 \\
\hline PREDICTED: dynein heavy chain 9, axonemal isoform X1 (H. sapiens) & 20.6 & 39 & $75 \%$ & 51 & $67 \%$ & $\mathrm{XP}_{0} 006721528.1$ \\
\hline dynein heavy chain 9 , axonemal isoform 2 (H. sapiens) & 20.6 & 52.4 & $75 \%$ & 51 & $67 \%$ & NP_001363.2 \\
\hline PREDICTED: proline-rich membrane anchor 1 isoform X1 (H. sapiens) & 20.2 & 20.2 & $66 \%$ & 71 & $75 \%$ & XP_006720107.1 \\
\hline Proline-rich membrane anchor 1 precursor (H. sapiens) & 20.2 & 20.2 & $66 \%$ & 71 & $75 \%$ & NP_821092.1 \\
\hline PREDICTED: WD repeat-containing protein 87-like isoform X4 (H. sapiens) & 20.2 & 106 & $83 \%$ & 72 & $86 \%$ & $\mathrm{XP}_{-005274478.1}$ \\
\hline PREDICTED: WD repeat-containing protein 87-like isoform X3 (H. sapiens) & 20.2 & 131 & $83 \%$ & 72 & $86 \%$ & XP_005274477.1 \\
\hline Uncharacterized protein loc100132994 (H. sapiens) & 20.2 & 20.2 & $41 \%$ & 72 & $100 \%$ & NP_001138611.1 \\
\hline PREDICTED: WD repeat-containing protein 87-like (H. sapiens) & 20.2 & 143 & $83 \%$ & 72 & $86 \%$ & $\mathrm{XP}_{-}^{-} 006726595.1$ \\
\hline PREDICTED: WD repeat-containing protein 87-like isoform X1 (H. sapiens) & 20.2 & 143 & $83 \%$ & 72 & $86 \%$ & $\mathrm{XP}_{-} 005274475.1$ \\
\hline protein FAM124A isoform 1 (H. sapiens) & 20.2 & 20.2 & $50 \%$ & 72 & $83 \%$ & NP_659456.3 \\
\hline Junctophilin-4 (Homo sapiens) & 20.2 & 20.2 & $75 \%$ & 72 & $70 \%$ & NP_115828.2 \\
\hline
\end{tabular}

Abbreviation: KCTD4, potassium channel tetramerization domain containing 4 . 


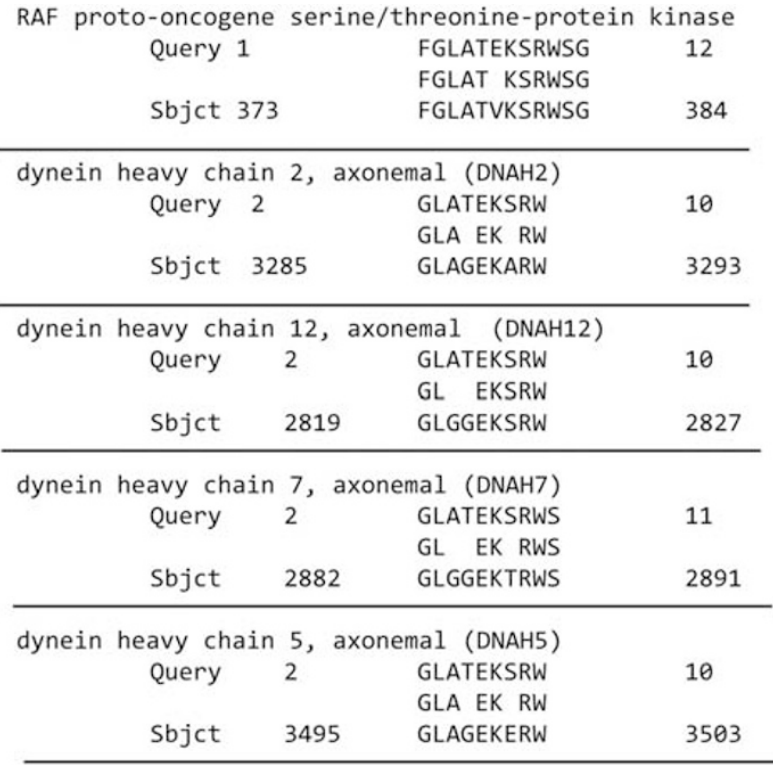

Figure 5 BLAST (Basic Local Alignment Search Tool) homology search of the human proteome using the sequence of the BRAF VE1 peptide immunogen (FGLATEKSRWSG). The regions of strongest identity between axonemal dynein heavy chain proteins and the BRAF V600E mutant epitope are shown.

cases and could therefore be a diagnostic pitfall particularly as the antibody gains increasingly wider acceptance and increased use. In addition, small biopsies, particularly those that have crush artifact or poorly oriented cells can also pose diagnostic difficulties. Strong staining of entrapped normal pituitary, as previously described ${ }^{15}$ and seen in most of the Rathke's cleft cyst specimens in this study, further adds to the complexity of analysis. Moreover, in tumors like ependymoma where cilia are found in microlumens and staining is therefore present in the cytoplasm, it is useful to recognize that this dot-like pattern of staining does not indicate the presence of an oncogenic mutation in $B R A F$ and is indeed providing similar diagnostic information as is garnered with epithelial membrane antigen staining. The BRAF V600E antibody has an important role in the evaluation of brain tumors such as pleomorphic xanthoastrocytoma and ganglioglioma, which frequently harbor mutations in $B R A F ; 1,13,17,18$ thus, understanding the sources of cross-reactivity of this antibody is of important clinical value.

A recent study has demonstrated that the VE1 antibody also stains a number of endocrine tissues, including the pituitary and the adrenal gland. ${ }^{19}$ There is preferential staining of discrete cell types with the strongest staining observed in adrenocorticotropic hormone (ACTH)-producing cells of the adenohypophysis and in cells comprising the inner segment of the zona fasciculata and the zona reticularis of the adrenal cortex. Accordingly, ACTH-expressing pituitary adenomas are stained with the VE1 antibody, yet these tumors do not
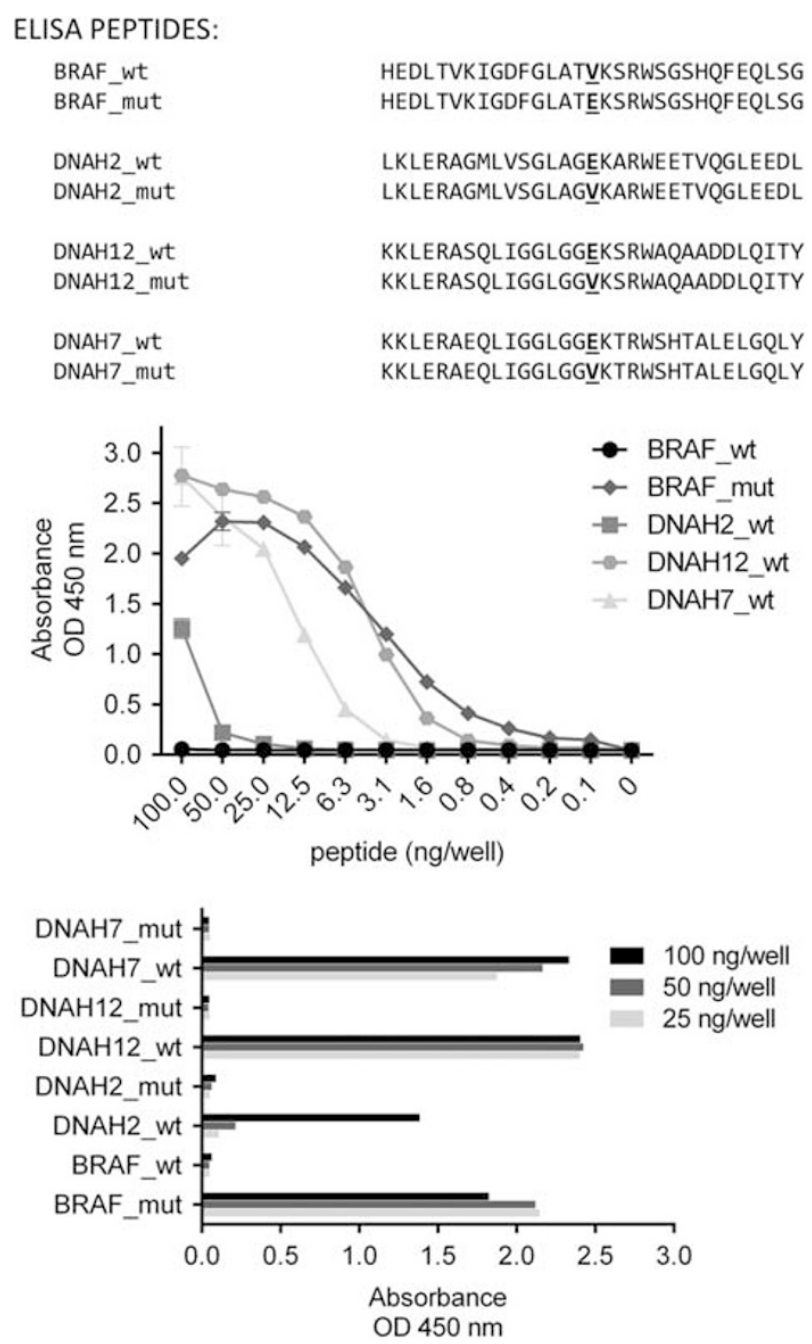

Figure 6 Enzyme-linked immunosorbent assay (ELISA) using the BRAF VE1 antibody and peptides from BRAF and axonemal dyneins. The peptides used in this experiment are displayed. ELISA details are explained in the Materials and methods section.

demonstrate detectable BRAF mutations. Using BLAST we see sequence similarities with proteins besides axonemal dynein heavy chains, some of which might be responsible for the cross-reactivity with endocrine tissues-such as $\alpha$-ketoglutaratedependent dioxygenase alkB homolog 7 , eukaryotic translation initiation factor $2-\alpha$ kinase 4 , polo-like kinase- $1 \delta$, potassium channel tetramerization domain containing 4, solute carrier family 4, anion exchanger, member 3 and armadillo repeat-containing protein 4.

In other tumors that harbor genetic changes of high clinical importance such as the EGFR L858R mutations in lung cancer ${ }^{20}$ and NRAS Q61R mutations in melanoma, mutation-specific antibodies can facilitate screening clinical cases. ${ }^{21,22}$ Antibodies that recognize other clinically relevant mutations such as the AKT1 E17K mutation that is found in breast cancer ${ }^{23}$ and in meningioma ${ }^{24}$ could also be of great use. During the use and validation 
of such antibodies, attention for potential crossreactivity by sequence homology searches could help identify potential cross-reactive proteins and corresponding tissues in advance.

\section{Acknowledgments}

We thank Terri Woo for assistance with immunohistochemistry, and Marian Slaney and Sebastian Valentin for assistance with histology. We thank Prathapan Thiru (Bioinformatics and Research Computing, Whitehead Institute for Biomedical Research) and Parker Merrill for assistance with bioinformatics analyses. This work was supported by the Jared Branfman Sunflowers for Life Fund for Pediatric Brain and Spinal Cancer Research, the V Foundation (SS) and by a grant from King Abdulaziz City for Science and Technology (KACST), Saudi Arabia (MAA).

\section{Disclosure/conflict of interest}

Sandro Santagata is a consultant and co-founder of Bayesian Diagnostics. All other authors declare no conflict of interests.

\section{References}

1 Capper D, Berghoff AS, Magerle M, et al. Immunohistochemical testing of BRAF V600E status in 1,120 tumor tissue samples of patients with brain metastases. Acta Neuropathol 2012;123:223-233.

2 Capper D, Preusser M, Habel A, et al. Assessment of BRAF V600E mutation status by immunohistochemistry with a mutation-specific monoclonal antibody. Acta Neuropathol 2011;122:11-19.

3 Kato Y, Jin G, Kuan CT, et al. A monoclonal antibody IMab-1 specifically recognizes IDH1R132H, the most common glioma-derived mutation. Biochem Biophys Res Commun 2009;390:547-551.

4 Capper D, Zentgraf $\mathrm{H}$, Balss J, et al. Monoclonal antibody specific for IDH1 R132H mutation. Acta Neuropathol 2009;118:599-601.

5 Preusser M, Capper D, Ilhan-Mutlu A, et al. Brain metastases: pathobiology and emerging targeted therapies. Acta Neuropathol 2012;123:205-222.

6 Preusser M, Capper D, Hartmann C, et al. testing in diagnostic neuropathology: review and practical guideline article invited by the Euro-CNS research committee. Clin Neuropathol 2011;30:217-230.

7 Chan JK, Ip YT, Cheuk W. The utility of immunohistochemistry for providing genetic information on tumors. Int J Surg Pathol 2013;21:455-475.

8 Santagata S, Eberlin LS, Norton I, et al. Intraoperative mass spectrometry mapping of an onco-metabolite to guide brain tumor surgery. Proc Natl Acad Sci USA 2014;111:11121-11126.
9 Brastianos PK, Taylor-Weiner A, Manley PE, et al. Exome sequencing identifies BRAF mutations in papillary craniopharyngiomas. Nat Genet 2014;46:161-165.

10 Voelker JL, Campbell RL, Muller J. Clinical, radiographic, and pathological features of symptomatic Rathke's cleft cysts. J Neurosurg 1991;74:535-544.

11 Shin JL, Asa SL, Woodhouse LJ, et al. Cystic lesions of the pituitary: clinicopathological features distinguishing craniopharyngioma, Rathke's cleft cyst, and arachnoid cyst. J Clin Endocrinol Metab 1999;84: 3972-3982.

12 Buslei R, Nolde M, Hofmann B, et al. Common mutations of beta-catenin in adamantinomatous craniopharyngiomas but not in other tumours originating from the sellar region. Acta Neuropathol 2005;109: 589-597.

13 MacConaill LE, Campbell CD, Kehoe SM, et al. Profiling critical cancer gene mutations in clinical tumor samples. PLoS One 2009;4:e7887.

14 Thiru P, Kern DM, McKinley KL, et al. Kinetochore genes are coordinately up-regulated in human tumors as part of a FoxM1-related cell division program. Mol Biol Cell 2014;25:1983-1994.

15 Mordes DA, Lynch K, Campbell S, et al. VE1 antibody immunoreactivity in normal anterior pituitary and adrenal cortex without detectable BRAF V600E mutations. Am J Clin Pathol 2014;141:811-815.

16 Altschul SF, Gish W, Miller W, et al. Basic local alignment search tool. J Mol Biol 1990;215:403-410.

17 Dias-Santagata D, Lam Q, Vernovsky K, et al. BRAF V600E mutations are common in pleomorphic xanthoastrocytoma: diagnostic and therapeutic implications. PLoS One 2011;6:e17948.

18 Lee EQ, Ruland S, LeBoeuf NR, et al. Successful treatment of a progressive BRAF V600E-mutated anaplastic pleomorphic xanthoastrocytoma with vemurafenib monotherapy. J Clin Oncol advance online publication, 4 August 2014; doi: 10.1200/jco. 2013.51.1766 (e-pub ahead of print).

19 Arisawa M, Makino T, McCann SM, et al. Effect of estrogen on the response of thyroid stimulating hormone to substance $\mathrm{P}$ in rats. Endocrinol Jpn 1989;36:899-903.

20 Mitsudomi T, Kosaka T, Endoh H, et al. Mutations of the epidermal growth factor receptor gene predict prolonged survival after gefitinib treatment in patients with non-small-cell lung cancer with postoperative recurrence. J Clin Oncol 2005;23:2513-2520.

$21 \mathrm{Yu}$ J, Kane S, Wu J, et al. Mutation-specific antibodies for the detection of EGFR mutations in non-small-cell lung cancer. Clin Cancer Res 2009;15:3023-3028.

22 Massi D, Simi L, Sensi E, et al. Immunohistochemistry is highly sensitive and specific for the detection of NRASQ61R mutation in melanoma. Mod Pathol advance online publication, 24 October 2014; doi: 10.1038/modpathol.2014.137 (e-pub ahead of print).

23 Carpten JD, Faber AL, Horn C, et al. A transforming mutation in the pleckstrin homology domain of AKT1 in cancer. Nature 2007;448:439-444.

24 Brastianos PK, Horowitz PM, Santagata S, et al. Genomic sequencing of meningiomas identifies oncogenic SMO and AKT1 mutations. Nat Genet 2013;45: 285-289.

Supplementary Information accompanies the paper on Modern Pathology website (http://www.nature.com/ modpathol) 